\section{T cell quorum sensing with Hippo}

The effector differentiation of $\mathrm{T}$ cells in response to infection must be carefully timed: too soon, and the effector population will be small; too late, and the infection may run out of control. In the Proceedings of the National Academy Sciences, Fearon and colleagues demonstrate a T cell-intrinsic mechanism by which these mutually exclusive imperatives of a $\mathrm{T}$ cell response can be controlled. Hippo signaling is a highly conserved developmental pathway that regulates organ size. The authors find that Hippo signaling is also triggered in a cell-cell contact-dependent manner after productive activation of T cells. After being activated, $T$ cells upregulate a pair of molecules, CTLA-4 and CD80; after their mutual interaction, this leads to assembly of the Hippo pathway and then to terminal differentiation. The frequency of such cell-cell interactions naturally increases after antigen-specific expansion and therefore leads to the optimally timed appearance of effector T cells.

Proc. Natl. Acad. Sci. USA (27 June 2012)

doi:10.1073/pnas.1209115109

\section{Suppressing glial activation}

Parkinson's disease is associated with neuroinflammation and the death of dopaminergic neurons. In Science Signaling, Hu et al. identify the kinase MEK1 as a target of the mitochondrial protease Omi. Omi is a stress-response protein that is released into the cytoplasm. Loss of Omi function is associated with neurodegeneration in Parkinson's disease in humans and in the mouse mnd 2 model. Mutation or knockdown of the gene encoding Omi in glial cells leads to greater abundance of MEK1 and phosphorylation of the downstream kinases Erk1 and Erk2, which is also observed in the brains of mnd 2 mice. This heightened Erk activation elicits enhanced production of inflammatory mediators, including TNF, IL-1 $\beta$ and reactive oxygen species. Supernatants from such Omi-deficient glial cells are toxic to primary neurons. Thus, the Omi protease normally acts as a brake to prevent excessive activation of the MEK1-Erk1/2 signaling pathway and diminish glial pro-inflammatory responses in the brain.

Sci. Signal. 5 (21 August 2012) doi: 10.1126/scisignal.2002946

$L A D$

\section{Leptin and mTor}

The adipocytokine leptin is associated with nutritional satiety, yet it also influences immune responses, as both leptin-deficient (ob/ob) mice and leptin receptor-deficient (db/db) mice are less prone to develop autoimmunity. In the Journal of Immunology, Procaccini et al. show that leptin signaling affects the axis of Akt and the nutrient sensor mTor in effector T cells. Leptin induces an autocrine feedback loop to activate PI(3)K-Akt-mTor, which leads to higher expression of leptin and its receptor. Inhibition of leptin signaling, even after ligation of $\mathrm{T}$ cell antigen receptor, diminishes the proliferation of effector $\mathrm{T}$ cells and IL-2 production. Conversely, leptin can partially restore $\mathrm{T}$ cell responsiveness in the presence of the inhibitor rapamycin. These findings confirm that nutritional status, as signaled via leptin production, directly intersects with the activation of effector $\mathrm{T}$ cells via the mTor pathway.

$L A D$

J. Immunol. (17 August 2012) doi:10.4049/jimmunol.120093

Written by Laurie A. Dempsey, Zoltan Fehervari \& Ioana Visan

\section{Anti-inflammatory signals}

Apoptotic cells express phosphatidylserine on the outer leaflet of the plasma membrane, which delivers an 'eat me' signal to phagocytes. In the Journal of Experimental Medicine, Nakahashi-Oda et al. show that CD300a, a transmembrane protein expressed by myeloid cells, binds phosphatidylserine on apoptotic cells but does not mediate phagocytosis by macrophages. Instead, after binding to phosphatidylserine, CD300a recruits the phosphatase SHP-1 and delivers an inhibitory signal, which results in suppression of the expression of cytokines and chemokines such as TNF, IL-13 and CCL2 in mast cells and macrophages. In a mouse model of peritonitis, enhanced chemokine secretion from CD300adeficient mast cells results in heightened recruitment of neutrophils to the peritoneal cavity and more-efficient bacterial clearance. These results indicate that apoptotic cells suppress inflammatory responses by mast cells and macrophages to help prevent excessive inflammation during bacterial infection.

J. Exp. Med. 209, 149-1503 (2012)

\section{Inflammation and wound repair}

Control of epithelia keratinocyte proliferation is an essential component of normal wound repair. In Immunity, Gallo and colleagues report that REG3A, an antimicrobial protein known to control bacterial proliferation in the intestine, is induced in keratinocytes after acute skin injury and contributes to wound healing by inhibiting differentiation and increasing the proliferation of keratinocytes. REG3A expression is induced via signaling by IL-17 through its receptor IL-17RA and promotes wound re-epithelization by binding to its receptor EXTL3, followed by activation of the kinases $\mathrm{PI}(3) \mathrm{K}$ and Akt. REG3A also has abundant expression in psoriatic lesions in the absence of acute injury in human patients and in mouse models of psoriasis. Because IL-17 also has abundant expression in psoriasis, the identification of REG3A as a stimulus for keratinocyte proliferation offers a link between IL-17-mediated inflammation and skin proliferation.

\section{Licensing the inflammasome}

Toll-like receptor 4 (TLR4) has a fundamental role in the response to Gram-negative bacteria. In Cell, Fitzgerald and colleagues identify a previously unknown pathway that contributes to the response to Gram-negative bacteria. Endosomal recognition of bacteria by TLR4 is mediated by the adaptor TRIF, and certain bacteria also trigger the proinflammatory cytokines IL- 1 and IL-18 via activation of the NLRP3 inflammasome. The authors find that TRIF is crucial for the response to NLRP3 triggering by Gram-negative bacteria but not by other bacteria or NLRP3 agonists. TRIF engagement typically results in the copious production of type I interferons (IFN- $\alpha$ and IFN- $\beta$ ), and these cytokines act in a feedback loop via the cognate receptor IFNAR to produce caspase-11. Although caspase- 11 is unable to process and mature the key inflammasome enzyme caspase-1, it is critical for 'licensing' NLRP3 activity. This TRIF-IFNAR-caspase-11 pathway may therefore be important in the uncontrolled inflammatory amplification seen in sepsis induced by Gram-negative bacteria.

Cell 150, 606-619 (2012) 